\title{
ASPECTOS POSITIVOS E NEGATIVOS DA ASSISTÊNCIA PRÉ-NATAL NO MUNICÍPIO DE RIBEIRÃO PRETO-SP*
}

Maria das Dores do Vale Oba** Maria Solange Guarino Tavares***

OBA, M.das D.do V.; TAVARES, M.S.G. Aspectos positivos e negativos da assistência pré-natal no município de Ribeirão Preto-SP. Rev.latino-am.enfermagem, Ribeirão Preto, v. 8, n. 2, p. 11-17, abril 2000.

O objetivo deste estudo foi identificar os aspectos positivos e negativos da assistência pré-natal na rede pública municipal de saúde de Ribeirão Preto-SP, na opinião de usuários e profissionais de saúde. Utiliza-se a abordagem qualitativa. Alguns avanços podem ser observados em relação à assistência pré-natal: a proximidade da Unidade de Saúde, ampliação da estrutura fisica, de recursos humanos e central de vagas na área da obstetrícia. Apesar destes aspectos positivos não foi possível ainda estabelecer a referência hospitalar e contra-referência nos diversos níveis do Sistema Único de Saúde. Evidencia-se uma desarticulação entre o período pré-natal, parto e puerpério.

UNITERMOS: saúde da mulher

\section{INTRODUÇÃO}

É com uma certa freqüência que os meios de comunicações nacionais relatam a precariedade da assistência prestada à mulher nas maternidades do país. Os problemas abordados vão desde imperícia e negligência médica, até condições estruturais referentes à organização dos serviços de saúde, para o atendimento à saúde da mulher.

Dados do Ministério da Saúde revelam que: “apenas 19\% dos estados brasileiros apresentam cobertura de pré-natal acima de 40\% e 9,7\% das mortes maternas no Brasil resultam de complicações decorrentes do aborto provocado. É inaceitável todo este descaso com assistência à mulher no periodo pré e perinatal, quando se sabe, que cerca de $60 \%$ da mortalidade infantil no país é devida a causas ligadas à gestação e ao parto..." (NOGUEIRA, 1994, p.17).

A assistência pré-natal, portanto, é um fator importante na redução da mortalidade materna e perinatal, visto que muitas patologias no período gravídico- puerperal podem ser tratadas e/ou controladas, evitandose efeitos danosos, já que um pré-natal de qualidade, certamente, orientará no sentido de se evitar problemas específicos do parto ou mesmo cuidados imediatos ao recém-nascido, além daqueles do período puerperal.

Embora o acompanhamento pré-natal já tenha provado sua eficácia na assistência a mãe e ao concepto, no que diz às taxas de morbi-mortalidade, ainda apresenta deficiência tanto na extensão da cobertura como no padrão de qualidade (MARCON,1997).

Observa-se um coeficiente de mortalidade materna no Brasil de 44,4 por 100.000 nascidos vivos, sendo as principais causas de morte materna: gravidez que termina em aborto $(10,1 \%)$, mortes maternas por causas obstétricas diretas $(73,1 \%)$ e mortes maternas por causas obstétricas indiretas (16,8\%) (BRASIL. MS.,1999).

Este fato, traduz a ineficácia dos serviços de saúde, quanto ao acesso, a freqüência, a qualidade da assistência pré-natal, parto e puerpério. No Estado de São Paulo em 1996, este coeficiente representou 41, 61 por

\footnotetext{
* Dados da Dissertação de mestrado: “Assistência pré-natal: uma assistência integral ou fragmentada?”, Escola de Enfermagem de Ribeirão Preto-USP(27/9/96), apoio financeiro do CNPq. Trabalho apresentado no $9^{\circ}$ Seminário Nacional de Pesquisa em Enfermagem/97

** Doutoranda do Programa Interunidades de Doutoramento em Enfermagem das Escolas de Enfermagem de Ribeirão Preto e São Paulo-Universidade de São Paulo, Mestre em Enfermagem de Saúde Pública pela Escola de Enfermagem de Ribeirão Preto da Universidade de São Paulo e especialista em: Enfermagem do Trabalho, Saúde Pública e Administração Hospitalar. Enfermeira e Gerente do Ambulatório Regional de Especialidade da SMS de Ribeirão Preto (N.G.A-59)

*** Professor Doutor em Enfermagem e Titular do Centro de Graduação em Enfermagem da Faculdade de Medicina do Triângulo Mineiro - Uberaba - Minas Gerais, orientadora
} 
100.000 nascidos vivos (SÃO PAULO, S. E. S., 1998).

O coeficiente de mortalidade materna de Ribeirão

Preto no ano de 1995 mostrou-se em 60,3 por 100.000 nascidos vivos, segundo dados do Comitê de Estudo e Prevenção da Morte Materna local. Nos países desenvolvido como o Canadá em 1990, este coeficiente apresentou 4 por 100.000 nascidos vivos (BRASIL. MS., 1994).

A assistência pré-natal em Ribeirão Preto vem sofrendo modificações em decorrência da política de saúde vigente, ou seja, um modelo assistencial de pronto atendimento, em que a assistência médica individual passou a ser a finalidade central da rede básica de saúde.

Observa-se um aumento da cobertura no pré-natal em Ribeirão Preto, mas o mesmo não pode ser dito quanto a qualidade do pré-natal. Dados do Comitê de Estudo e Prevenção da Morte Materna local de 1988 a 1995, ocorreram 43 mortes maternas, sendo 77\% decorrentes de causas obstétricas diretas, ou seja, hemorragia (26\%), hipertensão (14\%), aborto (10\%), puerpério(causas: infeção e hemorragia $(16 \%)$, outras causas $(11 \%)$ e causas obstétrica indireta (23\%) (OBA \& SILVA, 1997). Desta forma, questiona-se até que ponto a consulta pré-natal estaria identificando os fatores de risco e qual seria a relação estabelecida desta assistência com o parto e puerpério.

A mortalidade materna, conjuntamente com a mortalidade perinatal, traduz a eficácia dos serviços de saúde quanto a assistência pré-natal, parto e puerpério. Esperar-se que, quando melhor equipado e estruturado o serviço de saúde de um determinado local, menor os seus índices de mortalidade materna (SASS et al., 1995).

O relatório dos Comitês de Morte Materna do Paraná, de 1991, demostrou que, das 171 mulheres mortas em decorrências de causas obstétricas, $80,74 \%$ haviam comparecido a pelo menos uma consulta de pré-natal; encontrou-se uma média de cinco consultas por gravidez, sendo que, $43,47 \%$ das mulheres haviam comparecido a cinco ou mais consultas. Destas mortes, por sua vez, $28,14 \%$ ocorreram durante a gravidez, $8,38 \%$ durante o parto e $63,47 \%$ durante o puerpério (CARRANZA, 1994).

A morte materna nos países em desenvolvimento é como uma epidemia silenciosa, um problema de saúde pública, não só pela sua magnitude, mas porque mais de 90\% das causas de mortes, durante o ciclo gravidicopuerperal, são evitáveis (TANAKA,1995).

Assim, objetivou-se:

- identificar os aspectos positivos e negativos da assistência pré-natal na rede pública municipal de saúde de Ribeirão Preto-SP, na opinião de usuárias e profissionais de saúde.

\section{MEDOTOLOGIA}

Os dados deste estudo fazem parte da Dissertação de Mestrado: Assistência pré-natal: uma assistência integral ou fragmentada? (OBA, 1996), o qual foi apreciado pela comissão de ética do Centro Saúde Escola da Faculdade de Medicina de Ribeirão Preto-USP, realizado no mês de janeiro de 1996 em duas Unidades Distritais de Saúde, que prestam atendimentos de prénatal, localizadas na região central e oeste do município de Ribeirão Preto-SP.

Considerando o propósito da investigação, optouse pela abordagem qualitativa, uma vez que esta privilegia os sujeitos sociais, os quais detêm as informações que o investigador pretende conhecer (MINAYO, 1994). Elaborou-se uma questão norteadora: "Quais as vantagens e as desvantagens da assistência pré-natal desta unidade?". Os sujeitos sociais desta pesquisa foram: a clientela(sete gestantes) e os profissionais de saúde(três) de cada Unidade Básica Distrital de Saúde.

Como critério de inclusão optou-se pelo profissional e cliente, que participavam da assistência pré-natal no momento da investigação. Foram entrevistados 20 sujeitos sociais (um médico ginecologista, um enfermeiro, um auxiliar de enfermagem e sete gestantes de cada unidade), pois segundo MINAYO (1994) deve-se efetuar entrevistas em número suficiente, que permita uma certa reincidência de informações, assim como, a garantia de que estas contenham o conjunto das expressões dos vários elementos informantes.

Elegeu-se a técnica de entrevista semi-estruturada e análise documental (prontuários e manuais das Secretarias de Saúde Estadual e Municipal referentes a assistência pré-natal) como instrumento de coleta de dados. As entrevistas e as gravações foram realizadas após as autorizações dos sujeitos sociais e posteriormente transcritas. Tendo uma duração de trinta minutos cada entrevista.

O comportamento individual e social obedece a modelos culturais interiorizados, que refletem o caráter histórico e específico das relações sociais (OBA \& TAVARES, 1996).

Assim a questão norteadora fez vir à tona os aspectos positivos e negativos da assistência pré-natal, conforme relatos descritos a seguir.

Como pontos positivos da assistência pré-natal, segundo relatos das gestantes têm-se:

- a implantação da carteira de gestante; e

- o acompanhamento médico no pré-natal.

Como nesta fala a seguir:

V.P.J. 36 anos, multigesta, casada, primário completo,com renda familiar de quatro vezes ou mais o salário mínimo, do lar. 
"A carteira da gestante é única coisa deste pré-natal todo bonito do SUS, quando a gente vai, te leva para o médico no hospital, que está lá vê, é o único papel do pré-natal, Como eu disse o pré-natal é óóótimo, mas na hora de ter o nené é triste".

E como outros aspectos positivos da assistência pré-natal, segundo relatados das gestantes observa-se: - a realização e/ou referência para o exame de ultrasonografia; e

- a proximidade da Unidade Básica Distrital de Saúde da residência;

Tais aspectos são observados na fala a seguir:

V.P.J 36 anos, multigesta, casada, primário completo, com renda familiar de quatro vezes ou mais o salário mínimo, do lar. “... eu ainda sou de antigamente, a gente tinha que pega aquela filona do INPS de madrugada, agora o atendimento médico tá mais fácil, mais perto de casa.... Ah! agora eu faço aqui... é como se eu fosse em um consultório particular, o atendimento do médico foi como um particular, ele examinou,... explicou os exames, disse que tá tudo normal".

- a "referência" para o parto no Hospital das Clínicas da Faculdade de Medicina de Ribeirão Preto da Universidade de São Paulo.

Como nesta fala a seguir:

L.S.S. 26 anos, multigesta, casada, primário incompleto, com renda familiar de duas vezes o salário mínimo, do lar. "A gente sempre vai para o hospital. Da minha última foi na Beneficência, mas este eu vou te nas Clínicas. Eu sempre fui lá e sempre teve vaga, quando eu fiz aqui ...O parto, eles sempre manda para Clínicas...”.

Como pontos positivos da assistência pré-natal, segundo relatos dos profissionais de saúde têm-se:

- a existência de aparelho de ultra-sonografia;

- a existência do aparelho de cardiotocografia;

- a existência de laboratório de análise clínicas; e

- a central de vagas para internação na obstetrícia.

Nessa última década, o expressivo avanço tecnológico e a conseqüente incorporação passiva de equipamentos repercutiram de maneira sensível no processo de "reorganização da saúde". No setor da medicina neoliberal, devido às condições de financiamento e de custeio, foi possível a incorporação de tecnologia. Essa incorporação, associada à rápida expansão da medicina de grupo ou outras formas associativas de prestação de serviço e reforçada pela política neoliberal, trouxe a necessidade de "consumo" destas tecnologias e, conseqüentemente, a clínica foi relegada para segundo plano (TANAKA, 1995).

Pode-se confirmar essas observações na fala a seguir:

“....Aqui a gente tá em melhores condições em relação aos outros postos. $A$ gente tem um aparelho de ultra-sonografia, aparelho de cardiotocografia, laboratório de análise clínica no posto, que realiza exames de urina rotina, glicemia de jejum, tudo dentro do posto... O parto, com a central de vaga, tem melhorado muito este atendimento, eu oriento ela a procurar um hospital. E se lá não houver vaga, o próprio hospital liga para central e encaminha para onde tem vaga...".

Como outros aspectos positivos da assistência pré-natal, segundo relatos dos profissionais de saúde observa-se:

- a implantação da carteira de gestante;

- o acompanhamento médico no pré-natal; e

- a internação direta dos casos graves no Hospital das Clínicas da Faculdade de Medicina de Ribeirão Preto da Universidade de São Paulo.

Como nesta fala abaixo:

“... comparando com as outras unidades $e$ do que a gente conhece do trabalho aí fora, eu acho que o pré-natal é bem assistido, apesar de ser os doutorandos quem assistem a maioria do pré-natal, por conta da Dra......Mesmo escapando alguma coisa é bem fiscalizado, com controle de nutrição, de ultra-som. A gente checa muito na pós-consulta. Tem uma porção de unidades, que nem o exame preventivo faz. Então, eu acho que dentro do que eu conheço e como a gente segue o padrão do Hospital das Clínicas, é bem feito...".

"O acesso ao acompanhamento pré-natal é um indicador chave para avaliação do grau em que a saúde, como direito de todos e dever do Estado, se encontra numa determinada sociedade. Como também a prematuridade, a desnutrição e o baixo peso ao nascer refletem tanto a qualidade da atenção pré-natal e ao parto, quanto as condições de saúde e de nutrição da mãe, e, indiretamente, as condições de vida da população. Outros problemas de saúde que ocorrem durante a gravidez, como as infeções e as anemias, bem como as gestações freqüentes, o fumo, o uso de drogas e o desgaste no trabalho, seriam passiveis de solução através da assistência pré-natal"(NOGUEIRA, 1994, p.45).

As documentações oficiais da Secretaria de Estado da Saúde de São Paulo (SÃO PAULO, 1986) e Secretaria Municipal da Saúde de Ribeirão Preto 
(RIBEIRÃO PRETO, 1994) estabelecem como metas da assistência pré-natal:

- ampliar da cobertura e melhoria da qualidade das ações de pré-natal, parto e puerpério;

- matricular todas as gestantes da área programática da Unidade Básica de Saúde, para receber assistência prénatal;

- permitir o acesso fácil e seguro aos serviços de saúde; - promover ações de educação em saúde e debater sobre a condição feminina, nas Unidades de Saúde e em outras instituições;

- proporcionar as usuárias ações de assistência integral à sua saúde e informação sobre direitos e deveres para com o programa; e

- possibilitar aos profissionais envolvidos no programa: a participação efetiva na programação das atividades desenvolvidas no programa, as reciclagens periódicas e a realização de investigações clínicas.

Os depoimentos dos sujeitos sociais desta pesquisa, apresentam alguns pontos positivos da assistência pré-natal, mas estes avanços ainda estão muito aquém das diretrizes propostas pelos discursos oficiais.

Embora os profissionais de saúde e as gestantes valorizem a implantação da carteira de gestante, na pesquisa realizada por TANAKA (1995) demostrou, que a desarticulação entre os serviços de saúde leva a descontinuidade da assistência de pré-natal, parto e puerpério. Isso se agrava, uma vez que o médico no hospital dava pouca importância os registros contidos no cartão de pré- natal.

A desvalorização do registro é também extensiva ao profissional, que presta atendimento no pré-natal na unidade de saúde municipal de Ribeirão Preto, observase que este encontrava-se centrado na "incompreensibilidade", na "incompletude", ou seja, inexiste a informação referente anamnese geral e específica, exame físico geral e tocoginecológicos, caracterizando um atendimento mais ritualístico do que resolutivo (OBA \& TAVARES, 1998).

Os aspectos negativos da assistência pré-natal, segundo relatos das gestantes foram:

- o pré-natal feito por um médico e o parto por outro; e

- a inexistência de uma referência hospitalar.

Como nesta fala a seguir:

V.P.J. 36 anos, multigesta, casada, primário completo, com renda familiar de quatro vezes ou mais o salário mínimo, do lar. “O pré-natal é 100\% ótimo. Só é ruim, eu sou pobre, na hora de te o nené no hospital. O pré-natal sabe....em todas as unidades é ótimo. Eu fiz o outro no QuintinI foi ótimo também. Deveria ser pré-natal até na hora de te o parto, mas na hora de te é aquela loucura. Deveria te um encaminhamento para um local, para saber na hora de te. A gente faz um pré-natal e depois fica a Deus dará....".

A institucionalização do parto e o medo "histórico do mesmo" passam a ter outros componentes, como o medo do desconhecido, que traz à tona os receios de que, na grande maioria das vezes, quem realiza o prénatal não irá realizar o parto e haverá necessidade de mendigar, para se ter esta assistência durante o trabalho de parto (OBA \& TAVARES, 1996).

A gestante é obrigada a procurar por conta própria o local onde irá ter seu filho. Isso tem gerado ao longo do tempo, principalmente na última década, uma verdadeira peregrinação para se obter assistência hospitalar ao parto. Parece que cada serviço trabalha de modo estanque, como se a sua atividade tivesse um fim em si mesmo (TANAKA, 1995).

Como podemos observar nesta fala:

S.C.S. 24 anos, multigesta, casada,primário incompleto,com renda familiar de quatro vezes ou mais o salário mínimo, do lar.

“....No primeiro foi mais difícil, né? Eu estava com muita dor e fui no Hospital da Clínicas. Cheguei lá, não queria interna. Aí eu fiquei rodando em volta do hospital das 10:00 até 11:30 para dilata. Não internaram fui embora. Ai no outro dia, eu fui no postinho.O médico disse que já era para internar, já tava com quatro dedos de dilatação. Aí a minha irmã chamou o delegado, porque eles não queria internar. Aí ele examinou, aí estouro a bolsa. Ele internou. Antes dele examinar, ele tinha dito, para eu ir embora se eu fosse teria estourado a bolsa no caminho né".

Com a medicalização do parto após a sua institucionalização e a modernização da medicina, criou o saber médico na obstetrícia. A reorganização da assistência hospitalar acabou, com o passar do tempo, adquirindo uma visão mercantilista, em que o pobre paga com seu corpo, para que os médicos possam aprender e salvar (TANAKA, 1995).

Outros aspectos negativos na assistência prénatal, segundo relatos das gestantes foram:

- o rodízio médico durante assistência pré-natal.

Como nesta fala a seguir:

S.C.S. 24 anos, multigesta,casada,primário incompleto, com renda familiar de quatro vezes ou mais o salário mínimo, do lar. “Ah! No outro pré-natal eu passei por vários médicos e no parto foi outro. Não é tanto pelo médico do posto, mas eu achava que deveria ser um médico, no 
acompanhamento, né? Não é tanto no parto, mais no pré-natal, que deveria ser um só médico né?...".

A troca de médico a cada consulta de pré-natal não proporciona a mulher uma segurança desejada no transcorrer de sua gravidez. Esta insegurança é reforçada pelo, fato da gestante nem ter um médico certo na hora do parto, razão identificada como relevante para o início do acompanhamento do pré-natal (MARCON, 1997).

Aspectos negativos da assistência pré-natal, segundo relatos dos profissionais de saúde têm-se: - a informatização das agendas sem a identificação das atividades do programa de assistência integral à saúde da mulher;

Como podemos observar nesta fala:

“...ela tem que aguardar vaga na agenda médica um mês a dois meses. A agenda aqui é muito cheia ....ela fica de lá para cá se o médico do P. A. achar que não é urgência ela fica sem resolver o seu problema..."

"O problema sério que o computador trouxe foi, que o computador não diferencia gestantes de não gestantes, ela então fica esperam até dois meses para iniciar o pré - natal .Eu acho que deveria ter uma agenda separada, para gestante, mesmo porque, no final ela tem que vir mais freqüente e a gente tem que atender como eventual, como encaixe trabalhando além do limite de atendimento, para atender a paciente".

- a falta de treinamento para os profissionais;

- a demora nos resultados de exames de citologia e VDRL; - a desarticulação entre o período pré-natal , parto e puerpério;

- a inexistência de uma referência hospitalar; e

- a ausência de contra-referência.

Como observa-se nesta fala:

"Fomos adquirindo no contato do dia-adia aos trancos e barrancos. Vamos para um determinado posto e falaram: "Aqui você irá atender"....Só depois que fomos recebendo a planilha de protocolo é que ficamos sabendo o que a secretaria queria. E fomos descobrindo que tem ficha, para caso novo de obstetrícia, ginecologia, que tem o cartão de pré-natal. Uma das dificuldades que a gente nota, é a falta da contra-referência, por exemplo no pósparto. Outra desvantagem é a demora de alguns exames, por exemplo citologia vaginal tem demorado até três meses, VDRL tem demorado um pouco. $O$ que é às vezes é difícil de tratar aqui, você precisa encaminhar rapidamente, para dar resolução ao caso e não tem para onde encaminhar. Acho que é uma falha do sistema, talvez não próprio da UBS".

Outros aspectos negativos da assistência prénatal, segundo relatos dos profissionais de saúde foram: - um atendimento médico em forma de rodízio por estudantes e residentes de medicina.

Como observa-se nesta fala abaixo:

“Desvantagem é o rodízio de médico que não faz vínculo. Cada vez que ela vem é um é outro, né? A gente tem muitas dificuldade com algumas pacientes, por conta que os doutorandos trocam a cada três dias, nunca é o mesmo e, nestes três dias ela podem pegar um que não gosta de ginecologia de jeito nenhum. Então, ela sai de lá xingando, né? Porque realmente o atendimento pode ser muito seco e não aquela coisa, que a gestante está querendo conversar, né? pá-puffi e pronto! Então eu acho isto ruim...".

Os profissionais preocupam-se mais com a gravidez do que com a "mulher grávida", uma vez que todos os problemas/intercorrências apresentadas pelas mulheres são interpretadas como "normais", o que denota uma generalização dos sintomas sem se levar em consideração os aspectos individuais de cada mulher e de cada experiência de gravidez. Desta forma, não atende ás expectativas e necessidades reais destas (MARCON, 1997).

Isto é preocupante, pois neste serviço está sendo formado novos profissionais na área da medicina, e mesmo assim, não à devida atenção aos aspectos psicológicos, sociais, para subsidia-los na assistência junto as mulheres grávidas, de forma global e individualizada, o que sem dúvida poderá comprometer a assistência a ser prestada pelos mesmos em um futuro próximo (MARCON, 1997).

Como metas a ser alcançadas na assistência prénatal citada pelas documentações oficiais das Secretarias da Saúde de São Paulo e do Municipal de Ribeirão Preto (SÃO PAULO, 1986; RIBEIRÃO PRETO, 1994) temse:

- assegurar sistema de referência para atendimento, internações, exames laboratoriais e parto de gestante de alto risco;

- assegurar sistema de contra-referência para todas as gestantes garantindo consulta precoce no puerpério; - atuar nos hospitais da área com vista ao cumprimento das normas de alojamento conjunto;

- garantir atendimento na unidade básica de saúde ou em serviço de maior complexidade mediante referência, a todas as intercorrência específicas ou não da gestação; e 
- assistir à mulher através de um atendimento integral à saúde, com a finalidade de reduzir a morbidade e mortalidade das mulheres adolescentes e adultas residentes no Município de Ribeirão Preto.

Os depoimentos dos sujeitos sociais pontuam várias limitações da assistência pré-natal, que poderão refletir de forma direita ou indiretamente na saúde da mulher e esta mais uma vez poderá ser vitimada em conseqüência deste atendimento.

\section{CONSIDERAÇÕES FINAIS}

Alguns avanços podem ser apontados em relação à assistência pré-natal em Ribeirão Preto: a proximidade da Unidade Básica Distrital de Saúde, a ampliação da estrutura física e de recursos humanos, a aquisição de aparelho de ultra-sonografia e cardiotocografia, implantação da carteira de gestantes e da central de vagas para a internação na área da obstetrícia, e a internação direta no Hospital das Clínicas nos casos graves (OBA, 1996).

Estes avanços, foram possíveis, sem dúvida graças ao processo de descentralização desencadeado nos últimos dez anos, que segundo ALMEIDA et al. (1994) levou a gestão pública municipal da saúde a ter uma responsabilidade nova, uns com maiores possibilidades que outros, mas é inegável que houve uma ampliação da rede básica pública municipal, tanto na estrutura física com de recursos humanos. A distritalização do atendimento, de acordo com a complexidade e tecnológica. Assim como, uma maior participação da população nas políticas de saúde, através das comissões locais de saúde e do conselho municipal de saúde.

Apesar destes avanços na assistência pré-natal não foi possível ainda estabelecer a referência hospitalar e/ou a contra-referência nos diversos níveis do Sistema Único de Saúde de Ribeirão Preto. Evidencia-se uma desarticulação entre o período pré-natal, parto e puerpério.

O perfil das mulheres que utilizam estas unidades de saúde: baixa escolaridade e renda familiar e dependem do Sistema Único de Saúde, para o acompanhamento de pré-natal, resolução do seu parto e seguimento no puerpério e portanto mais propensas a morbi-mortalidade materna uma vez que esta sofre influência de fatores sócio-econômicos (OBA \& TAVARES, 1997).

As mulheres deparam-se com um atendimento médico individual, onde as consultas são ligadas basicamente nas queixas e condutas, à realização dos exames físicos, tocoginecológicos e à solicitação de exames complementares, não havendo tempo nem espaço para fala e/ou questionamento destas mulheres. Este atendimento é mais ritualístico do que preventivo ou resolutivo (OBA \& TAVARES, 1997).

Acredita-se que os sujeitos sociais envolvidos nesta assistência possam mudar a forma de fazer saúde, possibilitando uma prática na qual a equipe interdisciplinar apreenda a ouvir as necessidades das mulheres, que procuram a assistência pré-natal e em busca de vínculo, responsabilização e parceria na construção de uma verdadeira cidadania.

\section{POSITIVE AND NEGATIVE ASPECTS OF PRE NATAL CARE IN THE MUNICIPALITY OF RIBEIRÃO PRETO-SP}

This study had the purpose to identify the positive and negative aspects of pre natal care at the public health system of the city of Ribeirão Preto-SP, considering users and health professionals' opinions. Authors used a qualitative approach and found some improvements regarding pre-natal care such as: the proximity of the Health Unit, the improvement of physical and human resources structures and the opening of places for the obstetrics unit. Despite these positive aspects, authors were unable to find a hospital reference at the different levels of the Unified Health System. Results also showed a lack of articulation among prenatal, birth and puerperium periods.

\section{ASPECTOS POSITIVOS Y NEGATIVOS EN LA ASISTENCIA PRENATAL DEL MUNICIPIO} DE RIBEIRÃO PRETO-SP

Este estudio tuvo como objetivo identificar los aspectos positivos y negativos de la asistencia prenatal en la red pública municipal de Ribeirão Preto-SP en la opinión de paciente y profesionales, utilizando, para ello, el abordaje cualitativo. Algunos avances pueden ser observados en relación con la asistencia prenatal: la proximidad de la unidad de salud, ampliación de la estructura fisica y de recursos humanos e implementación de la central de cupos para la internación en la área de la obstetricia. A pesar de estos avances en la asistencia prenatal no fue posible establecer la remisión hospitalaria y la contra referencia en los niveles del Sistema Único de Salud de Ribeirão Preto. Se evidencia una desarticulacón entre asistencia prenatal, el parto y el puerpério. 


\section{REFERÊNCIAS BLIBIOGRÁFICAS}

01. ALMEIDA, M.C.P. et al. Gerência na rede básica de serviços de saúde em Ribeirão Preto-um processo em construção. Rev. Bras. Enfermagem, Brasília, v. 47, n. 3, p. 278-86, 1994.

02.BRASIL. Ministério da Saúde. Secretaria de Assistência à Saúde. Departamento de Programas de Saúde. Coordenação de Saúde Materno Infantil. Manual dos Comitês de mortalidade materna. Brasília, 1994.

03. .Ministério da Saúde. Programas de saúde da mulher/mortalidade materna.[on line] http:// datasus. saude.gov.br/cgi/tabcgi.exe?sim/ dxbr.def, 01/04/1999.

04.CARRANZA, M. Saúde reprodutiva da mulher brasileira. In: SAFFIOTI, H.I.B.; VARGAS, M.M. Mulher brasileira é assim. Rio de Janeiro: Rosa dos Tempos, 1994. p. 95-150.

05. MARCON, S.S. "Flashes" de como as gestantes percebem a assistência pré-natal em um hospital universitário. Rev.latino-am.Enfermagem, Ribeirão Preto, v. 5, n. 4, p. 43-54, 1997.

06. MINAYO, M.C.S. O desafio do conhecimento: pesquisa qualitativa em saúde. Rio de Janeiro: Hucitec-Abrasco, 1994.

07. NOGUEIRA, M.I. Assistência pré-natal: prática de saúde a serviço da vida. Rio de Janeiro: Hucitec, 1994.

08. OBA, M.D.V. Assistência pré-natal: uma assistência integral ou fragmentada?. Ribeirão Preto, 1996. Dissertação (Mestrado) - Escola de Enfermagem de Ribeirão Preto, Universidade de São Paulo.
09. ; SILVA, M.G. Mortalidade materna uma epidemia silenciosa no município de Ribeirão Preto-SP. /Trabalho apresentado no $49^{\circ}$ Congresso Brasileiro de Enfermagem, Belo Horizonte, 1997/ (mimeo).

10.___ ; TAVARES, M.S.G. As mulheres e os receios vivenciados em suas trajetórias obstétricas. Rev. Bras. Enfermagem, Brasília, v. 49, n .4, p. 56980, 1996.

11. ; TAVARES, M.S.G. Precariedade dos registros de assistência pré-natal em uma unidade Básica de saúde no município de Ribeirão PretoSP, Rev.latino-am.Enfermagem, Ribeirão Preto, v. 6, n. 1, p. 53-61, 1998.

12. RIBEIRÃO PRETO. Secretaria Municipal da Saúde. Coordenação do programa de assistência integral a saúde da mulher. Ribeirão Preto, 1994.

13. SÃO PAULO. Secretaria de Estado da Saúde de São Paulo, Grupo da Mulher. Subprograma de saúde da mulher: pré-natal normal. São Paulo, 1986. v. 2.

$14 . \quad$ Secretaria de Estado da Saúde de São Paulo. Fundação Sistema Estadual de Análise de Dados(SEADE). Mortalidade infantil e materna. [on line] http://www. deade.gov.br/egi-bin/ tiabp/shatp?sal/ANU96/sau960008-ANU96, 04/04/1998.

15. SASS, N. et al. Pré-natal a melhor arma na prevenção da mortalidade materna por hipertensão arterial. Femina, São Paulo, v. 23, p. 851-6, 1995.

16. TANAKA, A.C.d' A. Maternidade: dilema entre nascimento e morte. São Paulo: Hucitec, 1995. 